\title{
Current and Future Perspectives on Children's Genetic- and Endophenotype-Based Differential Susceptibility to Parenting
}

\author{
Karen Fischer ${ }^{1,2} \cdot$ Joyce Weeland ${ }^{1} \cdot$ Patty Leijten $^{1,2} \cdot$ Alithe van den Akker $^{1,2} \cdot$ Geertjan Overbeek $^{1,2}$
}

Published online: 3 October 2019

(c) The Author(s) 2019

\begin{abstract}
Objectives Accumulating research provides support for differential susceptibility, which holds that the same children who are most vulnerable to adversity, such as negative parenting, may also benefit most from enriched environments, such as positive parenting. This "for better and for worse" phenomenon is believed to be rooted in endogenous, biological susceptibility factors such as genes, and cognitive and physiological endophenotypes (e.g., heart rate variability and skin conductance). The goal of this paper is to discuss the effect of this biological perspective on children's susceptibility, and the inclusion of genetic and endophenotypical data in parenting research to shed light on the differential effects of parenting behavior We discuss a number of conceptual and methodological issues related to prior studies that have aimed to assess this.

Methods We review and discuss current and future perspectives on children's genetic- and endophenotype-based differential susceptibility to parenting, and experimental study designs that can adequately assess the within-person phenomenon of differential susceptibility.

Results We summarize our call for research in an experimental paradigm to test children's gene- and endophenotype-based differential susceptibility to parenting in their development of externalizing behavior.

Conclusions Hereby we aim to advance our understanding of the biological mechanisms underlying children's differential susceptibility to negative and positive parenting.
\end{abstract}

Keywords Experiment $\cdot$ Externalizing behavior $\cdot$ Differential susceptibility $\cdot$ Endophenotypes $\cdot$ Gene-environment interactions

Within the last decades, the differential susceptibility hypothesis (Belsky 1997), a within-person phenomenon that states that the same children who are most vulnerable to adversity may also benefit most from enriched environments, has received much scientific attention. The majority of research on this hypothesis has been conducted in the parenting context. The findings seem to indicate that children differ in the extent to which they benefit from improved parenting or suffer from dysfunctional parenting due to individual differences (Bakermans-Kranenburg and

Karen Fischer

k.fischer@uva.nl

1 Research Institute of Child Development and Education, University of Amsterdam, 1001NG Amsterdam, The Netherlands

2 Research Priority Area Yield, University of Amsterdam, 1001NG Amsterdam, The Netherlands
Van IJzendoorn 2015; Slagt et al. 2017). These individual differences "for better and for worse" are believed to be rooted in certain endogenous, biological factors such as genes and physiological endophenotypes (e.g., heart rate variability and skin conductance) (see for example Belsky and Pluess 2009; 2013; Boyce and Ellis 2005). Our goal in the present paper is to discuss the current and future perspectives on children's genetic- and endophenotype-based differential susceptibility to parenting in the development of externalizing behavior.

Meta-analytical evidence from $\mathrm{G} \times \mathrm{E}$ research supports that genetic predisposition (Van IJzendoorn and Bakermans-Kranenburg 2015; Bakermans-Kranenburg and Van IJzendoorn 2015) represents one such "for better and for worse" factor that makes children more susceptible to the development of externalizing behavior in the context of dysfunctional parenting but also more susceptible to prosocial development from positive parenting (Belsky and Pluess 2009, 2013; Boyce and Ellis 2005). Next to genetic 
susceptibility research, more research accumulated within the last decade on endophenotypes as susceptibility markers. Endophenotypes are genetically informed, stable biobehavioral traits that an individual is born with that function as mediators between genes and phenotype (Rommelse et al. 2011). Such intermediate phenoytpical traits can express themselves in different biological pathways, such as physiological markers of heart rate variability or skin conductance (El-Sheikh et al. 2001; El-Sheikh 2005), but also in information processing pathways such as cognitive biases (Fox and Beevers 2016). Endophenotypes seem to be of relevance to differential susceptibility research, because they are intrinsic traits that can be asssessed as measures of sensitivity to the environment, but are more proximal than genetic susceptibility and less invase to measure.

This biological perspective on children's susceptibility encourages the inclusion of genetic and endophenotypical data in parenting research to shed light onto how family environments shape children's differential susceptibility to future behavioral outcomes. However, the field is struggling with several conceptual and methodological issues. Firstly, the developmental process through which $\mathrm{G} \times \mathrm{E}$ affects children's externalizing behavior remains unclear. There is a lack of knowledge on what the mechanisms are through which genetic susceptibility operates, whereby more research is necessary that assesses genetic susceptibility together with other endophenotypical susceptibility markers jointly in one paradigm. Secondly, due to recent rapid advances in the field of behavioral genetics, there is a lively debate on how to best construct genetic indices that account for true genetic complexity (Dick 2018; Duncan et al. 2014). Third, although differential susceptibility is a withinsubjects phenomenon, prior studies have predominantly tested the differential susceptibility hypothesis with between-subjects designs, thus not providing a true test of differential susceptibility.

Therefore, the goal of this paper is to discuss in a first part (I) current and future perspectives on children's genetic- and endophenotype-based differential susceptibility to parenting. Regarding genetic differential susceptibility, we highlight the issue of replicability in $\mathrm{G} \times \mathrm{E}$ studies in the field of behavioral genetics. Notably, the review of literature in this paper is not meant to provide an exhaustive review of the GxE literature, but rather is meant to emphasize specific highlights and points of interest with regard to the issue of genetic and endophenotypic susceptibility. In a second part (II) we discuss study designs that enable true tests of children's differential susceptibility to parenting. In the last part, (III) we propose an experimental paradigm to test children's gene- and endophenotype-based differential susceptibility to parenting in their development of externalizing behavior. We propose a mediated-moderation model, specifying the effect of parenting on subsequent child behavior, its moderation by a polygenetic susceptibility index, and mediation of this moderation effect via endophenotypes in cognitive and physiological pathways. We highlight the importance of assessing these different biological susceptibility factors jointly within studies, in order to provide specific tests of interactions between biological dispositions and environmental (risk) factors. Our aim is to delineate an experimental research paradigm to test children's biologically based differential susceptibility to parenting in their development of externalizing behavior. In the long run, biologically-informed experimental research on children's differential susceptibility to parenting may advance our understanding of how externalizing child behavior develops and how parenting intervention programs could be tailored to the individual in the backdrop of their biological susceptibility.

\section{Part I: Current and Future Perspectives on Biologically Informed Measures of Children's Differential Susceptibility to Parenting}

\section{Genetic Susceptibility}

Since the intriguing finding by Caspi and colleagues (2002) that maltreated children with a genotype conferring high levels of expression of the Monoamine Oxidase A (MAOA) were less likely to develop antisocial problems than children without this genotype, there has been a fast growing literature on $\mathrm{G} \times \mathrm{E}$. These studies mostly focus on genetic polymorphisms that contribute to individual differences in the regulation of neurotransmitters such as dopamine (i.e., DAT1, DRD2, DRD4: Bakermans-Kranenburg and van IJzendoorn 2011), serotonin (i.e., 5-HTTLPR: Karg et al. 2011; Van IJzendoorn et al. 2012), and the degradation of dopamine, serotonin, and norepinephrine (i.e., MAOA: Byrd and Manuck 2014). Studies initially focused on single candidate genes, in which a single gene-marker is investigated (Maglione et al. 2018; Shi and Nehorai 2017; Weeland et al. 2015). The primary limitation of this approach finds itself in our lack of knowledge about etiological processes, especially in psychiatric disorders (Dick, Riley, \& Latendresse 2011). Therefore, there has been a growing call for an approach that accounts for genetic complexity (Dick et al. 2015).

In response, several recent studies have used cumulative polygenetic indices (e.g., Belsky and Beaver 2011; Chhangur et al. 2017) or examined specific haplotypes (e.g., $\mathrm{Li}$ et al. 2016). A polygenetic index integrates contributions of separate multiple common genetic variants, typically of small magnitude, predicting a specified outcome. Up to this date, these indices were mostly constructed in a way that they contained a handful of "usual suspect" markers or were 
broken down again into separate categories, specifying more versus less susceptible subgroups. Clearly, such an approach neither solves the problem of low statistical power, nor does it fully exploit all genetic information available. Ideally, polygenetic constructs contain genetic variants that are interrelated in some genetic pathway, with a known neurobiological function (Dick et al. 2015). This has however been difficult since we have limited knowledge about the exact function of many genetic polymorphisms in the development of children's reactivity. In summary, the candidate gene approach has evolved into research on polygenic indices to account for true genetic complexity, where we encountered new challenges, such as lack of knowledge about neurobiological functions and oversimplification in the construction of these indices. Given that polygenic indices up to this date predominantly inspect only a handful of single-nucleotide polymorphisms (SNPs), this approach can be at most considered an advanced version of a candidate-gene-driven approach.

In turn, these challenges have led to a genome-wideassociation (GWAS) approach, where several thousand SNPs are inspected throughout the entire genome rather than a pre-specified region or set of suspect polymorphisms. A recent example comes from a study by Keers et al. (2016) who, from a differential susceptibility standpoint, argued that genetic variability is not expected to directly associate with a developmental outcome, but rather through differences in individuals' reactivity to environmental stimuli. They used SNPs from a GWAS to predict differences in emotional problems between monozygotic twins, and constructed polygenetic indices based on this procedure-and these polygenetic indices were found to significantly moderate the association between parenting and emotional problems. Another recent study by Van Assche et al. (2017) genotyped 4947 SNPs in 263 genes, selected through a literature survey, in 982 adolescents of Caucasian origin. They assessed multiple parenting strategies (perceived parental psychological control, harsh punishment, and support) and found that two GABA-related genes (GABRR1 and GABRR2) interacted with parental support. In another approach by Nelemans et al. (2018) social anxiety was predicted by a polygenetic index that was constructed with a principal covariates regression technique (Vervloet et al. 2015). This technique allows researchers to create a weighted sum of SNPs, while simultaneously predicting an outcome measure. In the study by Nelemans et al. (2018), the selection of SNPs was biologically informed: all 223 SNPs belonged to an oxytocin pathway. The analysis yielded a genetic five-factor structure, with one putative genetic risk factor being strongly associated to social anxiety symptoms in adolescents, and significantly moderating the impact of both negative and positive parenting on adolescents' social anxiety. In summary, these different, promising approaches illustrate that there are several manners of how to construct genetic indices that take into account genetic complexity, and we are awaiting their replication in the future.

Despite the advances that have been made in the construction of genetic susceptibility indices, the missing heritability problem represents an ongoing issue of concern to all $\mathrm{G} \times \mathrm{E}$ studies in the behavioral and psychiatric genetics field. Studies that examine a genome-wide set of genetic variants or a psychopathological outcome are also not immune to this problem. Even for mental disorders that show the highest mapping of risk loci, identified risk variants make up only a small percentage of the heritability. Moreover, findings from GWAS are not always replicated (e.g., the findings on conduct disorder of Dick et al. 2011 vs. Anney et al. 2008). Recent data from five large population-based cohorts and three target samples with GWAS data suggests that externalizing behaviors in general are highly polygenic and have potential heterogeneous genetic effects across sex (Tielbeek et al. 2017). Thus, a crucial question still lying before us now is how we can take this complexity into account and identify replicable, reliable $\mathrm{G} \times \mathrm{E}$.

\section{Endophenotypes}

Even when addressing polygenetic susceptibility soundly, the developmental process through which $\mathrm{G} \times \mathrm{E}$ affects children's externalizing behavior remains unclear. A crucial question that remains is: how does genetic susceptibility work? The answer to this question might relate to the examination of endophenotypes: genetically informed, biobehavioral traits. These endophenotypes may determine how children for example react to emotions from parents and to parental punishment and reward cues in the socialization process (Weeland et al. 2015). For example, neurotransmitters may modulate reactivity to environmental stimuli and relate to a range of neurobiological processes underlying individuals' cognitions, mood, and motor control. For example, lower dopamine uptake after it is being released (related to, amongst others, COMT Val-allele, $M A O A$ high-activity allele, DRD4 7-repeat allele, DRD2 A1-allele, and DAT 10-repeat allele) has been associated with a blunted response to ordinary reward cues in the brain and low reward sensitivity (Pessiglione et al. 2006; Schultz 2016). Specifically, lower amounts of dopamine receptors might contribute to a reduced salience of positive environmental stimuli (specifically from delayed rewards), increased theta-beta ratio in resting state electroencephalography (EEG), and impaired reinforcement learning (Comings and Blum 2000; Massar et al. 2014). Thus, children with lower dopamine uptake might experience rewards as less salient and might therefore be less 
sensitive to reward-based socialization techniques such as praise or reward systems.

Such gene-based neurobiological mechanisms might be found in different systems, such as cognitive and physiological, and can be measured through brain activity (e.g., EEG and functional magnetic resonance imaging (FMRI)), our stress response system (e.g., (variability in) heart rate, levels of cortisol and (nor)adrenaline), and information processing. Regarding physiological markers, evidence indicates that children's heart rate variability (i.e., respiratory sinus arrhythmia, or RSA) may function as a "forbetter-for-worse" predictor of children's susceptibility to parenting. For example, RSA has been related to emotion regulation, and indirectly to behavioral control and psychopathology (Beauchaine and Thayer 2015). Specifically, children with a lower heart rate variability show more anxiety in high-conflict families, but significantly less anxiety in low-conflict families, when compared to children with higher variability (El-Sheikh et al. 2001). Similarly, children with high stress reactivity through RSA were found to be better adjusted in the context of low adversity, but poorer adjusted in the context of high adversity (Obradovic et al. 2011). Furthermore, skin conductance level response has shown to function as a vulnerability factor in the context of marital conflict and negative child outcomes (El-Sheikh 2005).

Regarding cognitive markers, children might be differentially susceptible to the development of cognitive biases to both negative and positive environmental contexts in a for-better-for-worse manner. Fox and Beevers (2016) reviewed evidence for such a 'cognitive bias' hypothesis, illustrating that environmental influences, either positive or negative, in combination with genetic susceptibility increase sensitivity to the environment and produce what they call 'toxic' or 'enhancing' cognitive biases, which in turn affect an individual's experience of wellbeing (Fox and Beevers 2016). Future research should aim to examine these endophenotypes jointly at these various pathways-cognitive and physiological-and investigate them as symbiotic, explanatory mechanisms underlying gene-by-parenting interactions in children's problem and prosocial behavior.

In sum, there is currently limited knowledge about the exact function of many genetic polymorphisms in the development of children's reactivity and the appropriate construction of genetic indices, and despite evidence from literature that children's susceptibility to environmental influences seems to be both gene- and endophenotypebased, these susceptibility factors have however been investigated mostly in separate lines of research. However, research indicates that the mechanisms through which genetic susceptibility operates are both physiological and cognitive in nature and most likely function interdependently to symbiotically modulate susceptibility to environmental influences in children and adolescents. We therefore suggest joint assessment of these biological susceptibility factors as a next important step in research on children's differential susceptibility to parenting. This is important, because these mediators might form a bridge between genetic research and intervention research, because they are more closely related to problem behavior and represent less invasive measures of individual differences in susceptibility compared to genetic susceptibility measures (Dick 2018).

\section{Part II: Study Designs that Enable True Tests of Children's Differential Susceptibility to Parenting}

After addressing biological susceptibility factors such as children's genetics and endophenotypes that may underlie the developmental process through which $\mathrm{G} \times \mathrm{E}$ affects children's externalizing behavior, the next crucial step is implementing a study design that enables a true tests of children's differential susceptibility to parenting. Here we first highlight the use of within-subjects designs and then the implementation of both a positive and negative environmental measure to provide a true test of the withinsubjects phenomenon of differential susceptibility.

\section{Within-Subjects Design}

Currently, most studies on children's differential susceptibility to parenting have been conducted with between-subjects designs. This means that the effects of a positive and negative environmental influence were assessed, but compared across susceptibility groups and not in terms of the magnitude of change in positive and negative outcome measures within each individual. As such, a between-subjects design does not allow to answer the specific research question of whether the same child who profits most from positive parenting would also suffer most from inadequate parenting. Given that differential susceptibility represents a within-subjects phenomenon, it is crucial that results from such betweensubjects designs are corroborated by results from withinsubjects designs.

One of the first experimental within-subjects-and thus true tests of differential susceptibility-assessed children's positive and negative affect and negative emotionality as a differential susceptibility factor by exposing children to both negative and positive feedback by parents in the form of puppet role-plays (Slagt et al. 2017). Specifically, they hypothesized that if differential susceptibility were to hold, that children high on negative emotionality, receiving positive as well as negative 
feedback would display greater changes in outcome measures (for-better: positive affect and prosocial intentions; for-worse: negative affect and antisocial intentions) than children scoring low on negative emotionality. This study found that negative emotionality did not moderate changes in positive or negative affect in reaction to negative and positive feedback (Slagt et al. 2017). The result did thus not support the differential susceptibility hypothesis.

\section{Negative Environmental Measures}

Although within-subjects designs are crucial, a potential problem arises when using them to assess parenting influences on children. Specifically, it is the ethical issue of having participants undergo a negative environment induction, to assess the "for-worse" end of the differential susceptibility spectrum. Due to this, studies in which actual parent-child interactions are examined within the framework of the differential susceptibility hypothesis are limited in that they only stimulate positive parenting cognitions and behavior, lacking "for worse" environmental stimuli. This is understandable, given that it is clearly unethical to experimentally induce family risk that potentially harms child development. However, in order to assess the "for-better-and-for-worse" phenomenon of differential susceptibility, we also need a measure of the "for-worse" part of the spectrum. One way to overcome this is to (over)sample families that by default display behavior that is considered a "for worse" condition. If families, for example, adopt harsh parenting techniques, especially in the light of stressful tasks, then the unmanipulated control condition can function as a "for worse" condition, eliminating the need to experimentally evoke this condition (Chhangur and Weeland et al. 2017). Even though it needs to be considered that intervention effects for at-risk or clinical family settings may merely produce a "less aversive" rather than a "for better" optimally functioning parenting environment, this design represents a good option to circumvent ethical issues associated with inducing negative parenting. Nevertheless, this solution still warrants careful consideration and caution about ways to minimize burden and to provide these families with adequate help.

\section{Part III: Proposed Research Paradigm}

To truly test differential susceptibility we propose a research paradigm which integrates susceptibility measures of several biological pathways in an experimental study that uses a within-subjects design, testing a child's susceptibility to both a positive and negative parenting environment. To test the negative parenting environment and circumvent respective ethical issues, we suggest to use at risk-samples which are expected to display greater proportions of dysfunctional parenting behaviors in response to a stressful tasks. We suggest to integrate polygenetic susceptibility markers and candidate endophenotypes (e.g., cognitive and physiological markers), which may mediate the relationship between genetic susceptibility and behavioral outcomes. Figure 1 shows our conceptual mediated-moderation model, specifying (a) the effect of positive ("for-better") and negative ("for-worse") parenting on subsequent child behavior, (b) its moderation by a polygenetic susceptibility index, and (c) mediation of this moderation effect via endophenotypes at cognitive and physiological pathways. Theoretically, these mediators might be more closely related to the problem behavior and possibly are easier, more specific, cheaper and less invasive measures of individual
Fig. 1 Conceptual model for testing $\mathrm{G} \times \mathrm{E}$ in a microtrial

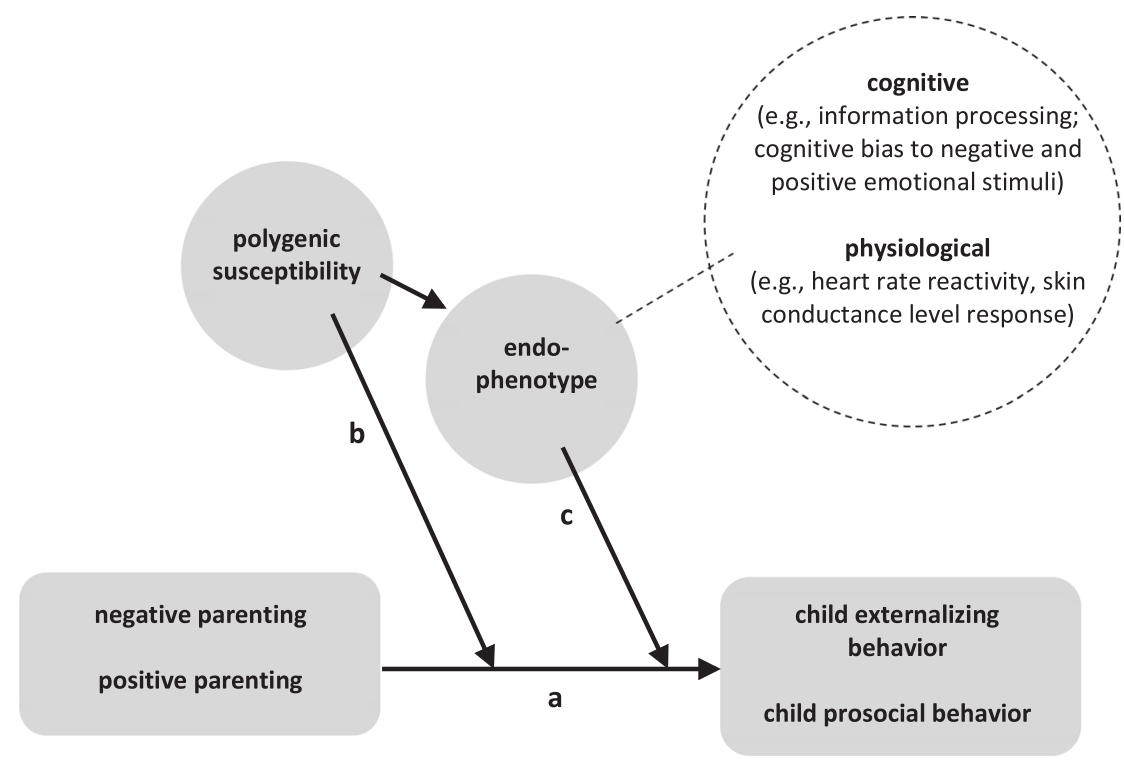


differences in susceptibility compared to genetic susceptibility measures (Dick 2018). More importantly, they might form a bridge between genetic research and intervention research, by enabling us to identify important differential targets for intervention programs. Indeed, in a recent study on polygenic scores previously associated with major depressive disorder, it was shown that in adolescents this association seemed to unfold via a combination of reduced parental monitoring and adolescent personality (Okbay et al. 2016).

\section{Conclusion}

Research on differential susceptibility has provided us with new insights in how genetic predisposition may express itself in endophenotypes that may explain why some children are more susceptible to both positive and negative environmental influences. However, the field faces challenges regarding the assessment of biological susceptibility and study designs that assess children's differential responses to parenting. In this paper, we aimed to discuss the current and future perspectives on children's geneticand endophenotype-based differential susceptibility to parenting in their development of externalizing behavior, and study designs that enable true tests of differential susceptibility. Lastly, we summarized our call for research in an experimental paradigm to test children's gene- and endophenotype-based differential susceptibility. Promising results have appeared in the past decade on children's biological susceptibility such as genetic predisposition, and physiological and cognitive endophenotype functioning. We hope to increase interest in assessing differential susceptibility measures using different biological pathways jointly in one paradigm, to explore the orchestrated effort of these pathways in determining children's susceptibility to parenting. Evident from this review is not only a demand for new approaches to construct genetic indices and a conceptualization of genetic susceptibility, but also the need for within-subjects studies that also include tests of the "forworse" end of the differential susceptibility framework, to provide a true test of differential susceptibility. In a successful scenario, clinical practice could benefit in terms of efficacy, cost-effectiveness, and personalized parenting intervention strategies.

Acknowledgements This research was supported by a Research Priority Area 'Yield' Graduate Programme grant 022.006.0 from the Netherlands Organisation of Scientific Research (NWO).

\section{Compliance with Ethical Standards}

Conflict of Interest The authors declare that they have no conflict of interest.
Publisher's note Springer Nature remains neutral with regard to jurisdictional claims in published maps and institutional affiliations.

Open Access This article is distributed under the terms of the Creative Commons Attribution 4.0 International License (http://crea tivecommons.org/licenses/by/4.0/), which permits unrestricted use, distribution, and reproduction in any medium, provided you give appropriate credit to the original author(s) and the source, provide a link to the Creative Commons license, and indicate if changes were made.

\section{References}

Anney, R. J., Lasky-Su, J., Ó'Dúshláine, C., Kenny, E., Neale, B. M., Mulligan, A., \& Arias-Vásquez, A. (2008). Conduct disorder and ADHD: evaluation of conduct problems as a categorical and quantitative trait in the international multicentre ADHD genetics study. American Journal of Medical Genetics Part B: Neuropsychiatric Genetics, 147, 1369-1378.

Bakermans-Kranenburg, M. J., \& van IJzendoorn, M. H. (2011). Differential susceptibility to rearing environment depending on dopamine-related genes: new evidence and a meta-analysis. Development and Psychopathology, 23, 39-52.

Bakermans-Kranenburg, M. J., \& Van IJzendoorn, M. H. (2015). The hidden efficacy of interventions: Gene $\times$ Environment interactions from a differential susceptibility perspective. Annual Review of Psychology, 66(11), 1-11. 29.

Beauchaine, T. P., \& Thayer, J. F. (2015). Heart rate variability as a transdiagnostic biomarker of psychopathology. International Journal of Psychophysiology, 98, 338-350.

Belsky, J. (1997). Variation in susceptibility to environmental influence: an evolutionary argument. Psychological Inquiry, 8, 182-186.

Belsky, J., \& Beaver, K. M. (2011). Cumulative genetic plasticity, parenting, and adolescent self-regulation. Journal of Child Psychology and Psychiatry, 52, 619-625.

Belsky, J., \& Pluess, M. (2009). Beyond diathesis stress: differential susceptibility to environmental influences. Psychological Bulletin, 135, 885-908.

Belsky, J., \& Pluess, M. (2013). Beyond risk, resilience, and dysregulation: phenotypic plasticity and human development. Development and Psychopathology, 25, 1243-1261.

Boyce, W. T., \& Ellis, B. J. (2005). Biological sensitivity to context: I. An evolutionary-developmental theory of the origins and functions of stress reactivity. Development and Psychopathology, 17, 271-301.

Byrd, A. L., \& Manuck, S. B. (2014). MAOA, childhood maltreatment, and antisocial behavior: meta-analysis of a geneenvironment interaction. Biological Psychiatry, 75, 9-17.

Caspi, A., McClay, J., Moffitt, T. E., Mill, J., Martin, J., Craig, I. W., \& Poulton, R. (2002). Role of genotype in the cycle of violence in maltreated children. Science, 297(5582), 851-854.

Chhangur, R. R., Weeland, J., Overbeek, G., Matthys, W., Castro, B., Giessen, D., \& Belsky, J. (2017). Genetic moderation of intervention efficacy: dopaminergic genes, the Incredible Years, and externalizing behavior in children. Child Development, 88, 796-811.

Comings, D. E., \& Blum, K. (2000). Reward deficiency syndrome: Genetic aspects of behavioral disorders. Progress in Brain Research, 126, 325-341.

Dick, D. M., Aliev, F., Krueger, R. F., Edwards, A., Agrawal, A., Lynskey, M., \& Almasy, L. (2011). Genome-wide association study of conduct disorder symptomatology. Molecular Psychiatry, 16, 800 .

Dick, D., Riley, B., \& Latendresse, S. J. (2011). Incorporating genetics into your studies: A guide for social scientists. Frontiers in Psychiatry, 2, 17. 
Dick, D. M., Agrawal, A., Keller, M. C., Adkins, A., Aliev, F., Monroe, S., \& Sher, K. J. (2015). Candidate gene-environment interaction research: reflections and recommendations. Perspectives on Psychological Science, 10(1), 37-59.

Dick, D. M. (2018). Commentary for special issue of prevention science "using genetics in prevention: Science fiction or science fact?". Prevention Science, 19, 101-108.

Duncan, L. E., Pollastri, A. R., \& Smoller, J. W. (2014). Mind the gap: Why many geneticists and psychological scientists have discrepant views about gene-environment interaction $(\mathrm{G} \times \mathrm{E})$ research. American Psychologist, 69, 249.

El-Sheikh, M., Harger, J., \& Whitson, S. M. (2001). Exposure to interparental conflict and children's adjustment and physical health: the moderating role of vagal tone. Child Development, 72 , 1617-1636.

El-Sheikh, M. (2005). The role of emotional responses and physiological reactivity in the marital conflict-child functioning link. Journal of Child Psychology and Psychiatry, 46, 1191-1199.

Fox, E., \& Beevers, C. G. (2016). Differential sensitivity to the environment: contribution of cognitive biases and genes to psychological wellbeing. Molecular Psychiatry, 21, 1657-1662.

Karg, K., Burmeister, M., Shedden, K., \& Sen, S. (2011). The serotonin transporter promoter variant (5HTTLPR), stress, and depression meta-analysis revisited. Archives of General Psychiatry, 68, 444-454.

Keers, R., Coleman, J. R., Lester, K. J., Roberts, S., Breen, G., Thastum, M., \& Eley, T. C. (2016). A genome-wide test of the differential susceptibility hypothesis reveals a genetic predictor of differential response to psychological treatments for child anxiety disorders. Psychotherapy and Psychosomatics, 85, 146-158.

Li, Y., Sulik, M. J., Eisenberg, N., Spinrad, T. L., Lemery-Chalfant, K., Stover, D. A., \& Verrelli, B. C. (2016). Predicting childhood effortful control from interactions between early parenting quality and children's dopamine transporter gene haplotypes. Development and Psychopathology, 28, 199-212.

Maglione, D., Caputi, M., Moretti, B., \& Scaini, S. (2018). Psychopathological consequences of maltreatment among children and adolescents: a systematic review of the GxE literature. Research in Developmental Disabilities, 82, 53-66.

Massar, S. A., Kenemans, J. L., \& Schutter, D. J. (2014). Resting-state EEG theta activity and risk learning: sensitivity to reward or punishment? International Journal of Psychophysiology, 91, 172-177.

Nelemans, S. A., Van Assche, E., Bijttebier, P., Colpin, H., Van Leeuwen, K., Verschueren, K., \& Goossens, L. (2018). Parenting interacts with oxytocin polymorphisms to predict adolescent social anxiety symptom development: a novel polygenic approach. Journal of Abnormal Child Psychology, 47, 1-14.

Obradovic, J., Bush, N. R., \& Boyce, T. (2011). The interactive effect of marital conflict and stress reactivity on externalizing and internalizing symptoms: the role of laboratory stressors. Development and Psychopathology, 23, 101-114.

Okbay, A., Baselmans, B. M., DeNeve, J. E., Turley, P., Nivard, M. G., Fontana, M. A., \& Cesarini, D. (2016). Genetic variants associated with subjective well-being, depressive symptoms, and neuroticism identified through genomewide analyses. Nature Genetics, 48, 624-633.

Pessiglione, M., Seymour, B., Flandin, G., Dolan, R. J., \& Frith, C. D. (2006). Dopamine-dependent prediction errors underpin rewardseeking behaviour in humans. Nature, 442, 1042.

Rommelse, N. N., Geurts, H. M., Franke, B., Buitelaar, J. K., \& Hartman, C. A. (2011). A review on cognitive and brain endophenotypes that may be common in autism spectrum disorder and attention-deficit/hyperactivity disorder and facilitate the search for pleiotropic genes. Neuroscience \& Biobehavioral Reviews, 35 (6), 1363-1396.

Schultz, W. (2016). Dopamine reward prediction-error signalling: a two-component response. Nature Reviews Neuroscience, 17, 183.

Shi, G., \& Nehorai, A. (2017). Robustness of meta-analyses in finding gene $\times$ environment interactions. PloS ONE, 12, e 0171446

Slagt, M., Dubas, J. S., van Aken, M. A., Ellis, B. J., \& Deković, M. (2017). Children's differential susceptibility to parenting: An experimental test of "for better and for worse". Journal of Experimental Child Psychology, 154, 78-97.

Tielbeek, J. J., Johansson, A., Polderman, T. J., Rautiainen, M. R., Jansen, P., Taylor, M., \& Viding, E. (2017). Genome-wide association studies of a broad spectrum of antisocial behavior. JAMA Psychiatry, 74, 1242-1250.

Van Assche, E., Moons, T., Cinar, O., Viechtbauer, W., Oldehinkel, A. J., Van Leeuwen, K., \& Goossens, L. (2017). Gene-based interaction analysis shows GABA ergic genes interacting with parenting in adolescent depressive symptoms. Journal of Child Psychology and Psychiatry, 58(12), 1301-1309.

Van IJzendoorn, M. H., Belsky, J., \& Bakermans-Kranenburg, M. J. (2012). Serotonin transporter genotype 5HTTLPR as a marker of differential susceptibility? A meta-analysis of child and adolescent gene-by-environment studies. Translational Psychiatry, 2, e147.

Van IJzendoorn, M. H., \& Bakermans-Kranenburg, M. J. (2015). Genetic differential susceptibility on trial: meta-analytic support from randomized controlled experiments. Development and Psychopathology, 27, 151-162.

Vervloet, M., Kiers, H. A., Van den Noortgate, W., \& Ceulemans, E. (2015). PCovR: an R package for principal covariates regression. Journal of Statistical Software, 65, 1-14.

Weeland, J., Overbeek, G., Orobio de Castro, B., \& Matthys, W. (2015). Underlying mechanisms of gene-environment interactions in externalizing behavior: a systematic review and search for theoretical mechanisms. Clinical Child and Family Psychology Review, 18, 413-442. 